\title{
HUGUES CHOPLIN (Compiègne)
}

\author{
Un art du quotidien ? \\ A partir de Georges Perec et Vilhelm Hammershøi
}

\author{
An art of the everyday? \\ Parting from Georges Perec and Vilhelm Hammershøi
}

\begin{abstract}
Contemporary continental philosophy seems to be deeply connected to art, whether it is poetry and literature (from Heidegger to Derrida), painting (Merleau-Ponty, Maldiney) or music (since Bergson). This connection is built upon the primacy of a trial of the invisible, which is very often a trial of the event. The aim of this article is to establish an art of the everyday freed from this contemporary "eventism". We thus examine how the researches of Georges Perec in literature and of Vilhelm Hammersh $\phi i$ in painting meditate on the strangeness of the everyday - its flatness, its silence - which are irreducible to this aesthetic trial as it can be conceptualized by the philosophy of the life of DeleuzeGuattari and by the humanism of Levinas. This research thus beckons towards an art of the common contesting the contemporary primacy of the vital and the (in)human.
\end{abstract}

Keywords: Vilhem Hammershøi, Georges Perec, art of the everyday, common, event

\section{Introduction}

Etablir un art du quotidien, affranchi du couple que la philosophie continentale contemporaine constitue avec l'art : tel est l'enjeu qui porte cet article.

Depuis Bergson et Heidegger au moins, la philosophie continentale (notamment française et allemande) semble connectée, de manière déterminante, à l'art. Que l'on songe au poids que la poésie, la littérature ou la question de l'écriture revêtent de Heidegger à Derrida, à l'ancrage de certaines phénoménologies (Merleau-Ponty, Maldiney) dans la peinture ou bien encore aux analyses que la philosophie française du mouvement vital (Bergson, Jankélévitch, Deleuze) consacre à la musique. Tout se passe comme si l'art désignait une condition (Badiou, 1992) de la pensée philosophique, comme si le déploiement de celle-ci nécessitait un passage par l'art, peut-être comme elle nécessitait, chez Platon ou Descartes, un passage par la géométrie. 
Ce couple contemporain de la philosophie et de l'art semble contenu par un motif : l'épreuve de l'invisible. Cette épreuve prend bien entendu des formes très diverses selon les philosophies de l'art considérées. Mais, aussi hétérogènes soient-elles, ces philosophies semblent bien toutes, en effet, établir comme ressort de l'art un affect (un éprouver) procédant d'une dimension qui excède l'expérience du visible (ou du sensible). Qu'il nous suffise ici de rappeler combien dès Husserl, l'expérience du visible est irréductiblement débordée par un rapport à l'invisible, qui annonce déjà, à sa manière, le "tournant esthétique de la phénoménologie" (Saison 1999, 125). Ou encore combien, chez Deleuze et Guattari, l'art a vocation à "rendre sensibles les forces insensibles [...] qui nous affectent" (nous soulignons, Deleuze et Guattari 1991, 172).

Epreuve de l'invisible : nous soutenons que ce motif contemporain, aussi important soit-il, ne saurait épuiser les recherches artistiques susceptibles de requérir singulièrement la pensée. Autrement dit, d'autres voies esthétiques, affranchies de ce motif, sont aujourd'hui à explorer. Nous proposons ainsi de nous tourner vers des artistes questionnant le quotidien, à savoir, selon Blanchot, "ce qu'il y a de plus difficile à découvrir" (Blanchot 2004, 355). Cette énigme du quotidien ne déjoue-t-elle pas en effet le primat de l'épreuve de l'invisible dans la mesure où, dans la pensée contemporaine - en phénoménologie comme dans la philosophie française du mouvement -, cette épreuve s'avère bien souvent être une épreuve de l'événement?

Deux recherches seront ici examinées : celles, en littérature, du français Georges Perec (1936-1982) et, en peinture, du danois Vilhem Hammershøi (1864-1916). Toutes deux invitent à méditer l'étrangeté du quotidien - sa platitude, son silence - en tant qu'ils se refusent à toute événementialité. En quel sens ces recherches peuvent-elles désigner un art du quotidien - ou peut-être un art du commun -, dépris de l'épreuve esthétique contemporaine de l'invisible?

\section{La platitude du quotidien. A partir de Perec}

\subsection{L'énigme Perec}

\section{L'inventaire du quotidien}

"Comment regarder le quotidien" (Perec 2003, 10), "comment en rendre compte, comment l'interroger, comment le décrire ?" (Perec 1989, 11) : tel est l'un des questionnements directeurs du travail littéraire de Perec. Ce dernier y répond en opérant un épuisement singulier du quotidien : "Se forcer à épuiser le sujet, même si ça a l'air grotesque, ou futile, 
ou stupide." (Perec 1976, 76). Cet épuisement passe, comme le dit Maurice Olender, par un "inventaire du quotidien" (Perec 1989, 7) : "Au début, on ne peut qu'essayer de nommer les choses, une à une, platement, les énumérer, les dénombrer, de la manière la plus banale possible, de la manière la plus précise possible, en essayant de ne rien oublier." (Perec 2019, 45). Par exemple: "Décrivez votre rue. Décrivez-en une autre. Comparez. Faites l'inventaire de vos poches, de votre sac. Interrogez-vous sur la provenance, l'usage et le devenir de chacun des objets que vous en retirez. Questionnez vos petites cuillers. Qu'y a-til sous votre papier peint ?" (Perec 1989, 12-13). L'Infra-ordinaire réunit ainsi plusieurs textes perecquiens déployant cette démarche d'épuisement ou d'inventaire sur différentes réalités quotidiennes : une rue de l'enfance de Perec ("La rue Vilin"), son bureau ("Still life/Style leaf") ou les aliments qu'il a mangés en 1975 ("Tentative d'inventaire des aliments liquides et solides que j'ai ingurgités au cours de l'année mil neuf cent soixantequatorze").

L'ouvrage dans lequel Perec déploie le plus significativement cette démarche est probablement Tentative d'épuisement d'un lieu parisien (1975). Ce texte est le produit d'une expérimentation conduite place St Sulpice, à Paris, lieu où, pendant trois jours, Perec s'attache à décrire "ce qui se passe quand il ne se passe rien, sinon du temps, des gens, des voitures et des nuages." (nous soulignons, Perec 2020, 10). Concrètement, le livre s'organise en neuf séquences, chacune de ces séquences correspondant à une phase du travail de Perec en temps réel, place St Sulpice. Dans chacune de ces phases - d'une durée comprise entre une petite heure et deux heures et demie -, Perec s'emploie à établir l'inventaire de ce qui (se) passe, tel qu'il peut l'observer depuis son lieu d'écriture dans cette place (un tabac, un café ou un banc).

Par exemple :

Passe une dame qui vient d'acheter un bougeoir moche

Passe un petit car : Club Resein Keller.

Car, Japonais. (ibid., 52).

Ou encore (à la toute fin du texte) : "Les pigeons sont sur le terre-plein. Ils s'envolent tous en même temps. Quatre enfants. Un chien. Un petit rayon de soleil. Le 96. Il est deux heures." (ibid., 65).

Si la description de ce qui (se) passe est en effet incontestablement le discours dominant de ce texte, elle s'y combine parfois avec d'autres types de discours :

- des tentatives de classification/catégorisation de type presque sociologique, qui peuvent s'opérer au début de certaines séquences du travail de Perec. Très vite, ces catégories plutôt générales (par exemple : "discussions à deux, discussions à trois, discussions à plusieurs 
[...] mode de locomotion : marche, véhicule", ibid., 17) sont débordées par des passages singuliers ou des "occurrence(s) singulière(s)" (nous soulignons, Schilling 2006, 123) : "un 96 passe. Un 87 passe. Un 86 passe. Un 70 passe. Un camion 'Grenelle Interlinge' passe." (Perec 2020, 19);

- des descriptions portant sur Perec lui-même en tant qu'il est engagé dans cette expérimentation. Par exemple : "j’ai froid, je commande un vieux marc" (ibid., 30) ;

- des commentaires de type conceptuel ou méthodologique concernant le quotidien. Par exemple : "comment voir le tissu si ce sont seulement les déchirures qui le font apparaitre : personne ne voit jamais passer les autobus, sauf s'il en attend un" (ibid., 48).

\section{Ni ordinaire, ni événementiel}

L'interprétation de tels textes est loin d'aller de soi. Pourquoi donc importe-t-il ainsi de "se forcer à écrire ce qui n'a pas d'intérêt, ce qui est le plus évident, le plus commun, le plus terne." (Perec 1976, 76) ? Pourquoi donc passer ainsi, par exemple, trois jours place St Sulpice à décrire "ce qui se passe quand il ne se passe rien..." ? Incontestablement, Perec veut ainsi donner accès à un autre niveau de notre existence : le reste. "... le reste : ce que l'on ne note généralement pas, ce qui ne se remarque pas, ce qui n'a pas d'importance" (Perec 2020, 10), ce qui, par conséquent, échappe aux journaux (et aux journalistes), lesquels en effet, "parlent de tout, sauf du journalier" (Perec 1989, 10). Autrement dit, épuiser un lieu, c'est "débusquer" "ces 'choses communes'" constitutives du reste et "les arracher à la gangue dans laquelle" elles sont "engluées" (ibid., 11). Mais comment donc comprendre précisément cet "arrachement" des choses communes et du reste?

Certains lecteurs de Perec considèrent que son travail consiste à dévoiler la "richesse insoupçonnée du quotidien" (Schilling 2006, 127). Une telle lecture peut rapprocher Perec d'un auteur comme Michel de Certeau qui, à la même époque, soulignait, dans L'Invention $d u$ quotidien, combien l'homme ordinaire invente le quotidien en résistant aux normes, aux disciplines du pouvoir (au sens de Foucault) et à l'ordre social - par exemple à cet ordre propre à l'espace organisé d'une ville, que déjouent les pratiques ordinaires de marche. De ce point de vue, le reste du quotidien instituerait le registre de l'ordinaire en tant qu'il résiste au normal, et, peut-être, aux maladies dont celui-ci est porteur (Le Blanc, 2007).

Incontestablement, certains textes de Perec donnent prise à une telle lecture, en particulier Espèces d'espaces, dont le sous-titre, significatif, est Journal d'un usager de l'espace. Mais les inventaires du quotidien perecquiens - et en particulier Tentative d'épuisement... ne font nullement valoir une contestation inventive des normes du social. Les descriptions de Perec font bien état d'une profusion d'occurrences irréductibles aux classifications et 
catégories qu'il tente lui-même d'établir parfois (par exemple au début de certaines de ces séquences de travail dans Tentative d'épuisement...). En ce sens, cette profusion excède sans doute les grilles du normal. Pour autant, cet inventaire du quotidien n'établit aucune résistance ou inventivité propre à l'homme ordinaire. De quelle résistance ou inventivité ordinaire témoignerait donc, par exemple, le passage de cette jeune fille : "Passe une jeune fille ; elle porte une raquette de tennis sous le bras (dans une housse en tissu où l'on peut aussi ranger les balles)" (Perec 2020,49) ? Ni inventivité, ni contestation : il est tout à fait remarquable de ce point de vue que, dans son travail place St Sulpice, Perec s'avère comme indifférent aux rapports sociaux qui, pourtant, s'attestent inévitablement dans un tel lieu ou bien encore qu'il ne relève pas "ces milliers de faits de la vie quotidienne touchant au racisme, au sexisme ou à l'aliénation économique" (Schilling 2006, 124). Comme le soutient Derek Schilling, à la différence de Certeau, "Perec se contente de rendre sèchement compte d'actes et de gestes impersonnels (garer une voiture, traverser la rue...), d'itinéraires et d'emplois du temps [...]. Ce quotidien-là [...] est répétitif plutôt qu'inventif" (nous soulignons, ibid., 181) - ou encore, dirons-nous, insistant plutôt que résistant. Comme si, étrangement, cette insistance du quotidien - "qui passe chaque jour et revient chaque jour" (Perec 1989, 21) - déjouait le normal et l'ordre social sans pour autant y résister ou les contester...

Que le quotidien de Perec ne soit ni normal, ni ordinaire, c'est ce que confirme la dimension spatiale pour le moins étrange qu'il engage. L'inventaire du quotidien doit en effet :

Continuer

jusqu'à ce que le lieu devienne improbable,

jusqu'à ressentir pendant un très bref instant l'impression d'être dans une ville étrangère, ou, mieux encore, jusqu'à ne plus comprendre ce qui se passe ou ne se passe pas, que le lieu tout entier devienne étranger, que l'on ne sache même plus que ça s'appelle une ville, une rue, des immeubles, des trottoirs... (Perec 1976, 80)1.

Comment donc penser ce "devenir étranger" auquel conduit le travail d'épuisement d'un lieu quotidien ? Procède-t-il d'une forme de vide - ou d'"extase du vide" (Yvan 2007, 143) - que Perec suggère en effet, à un moment, dans Tentative d'épuisement... :

${ }^{1}$ Perec écrit ainsi, à la fin de Tentative d'épuisement... : "En ne regardant qu'un seul détail [...], on peut, sans aucune difficulté, s'imaginer que l'on est à Etampes ou à Bourges, ou même quelque part à Vienne (Autriche) où je n'ai d'ailleurs jamais été." (Perec 2020, 64). 
"Instants de vide" (Perec 2020, 59), et surtout, à la toute fin d'Espèces d'espaces : "Ecrire : [...] arracher quelques bribes précises au vide qui se creuse" (Perec 1976, 141) ?

Il semble difficile ici de ne pas mentionner l'histoire personnelle de Perec et tout particulièrement la déportation de sa mère à Auschwitz. Sa recherche sur le quotidien, "dont le caractère" est "à première vue artisanal", comporterait ainsi une dimension dramatique et même une "portée métaphysique" (Macherey 2005), qui ne font que renforcer la difficulté d'analyse de ces textes. Comment donc articuler, en effet, l'inventaire du quotidien et cette dimension "métaphysique" ? Une piste serait de convoquer le ressort contemporain de l'événement. L'écriture de Perec serait-elle en effet fortement affectée par l'événement d'Auschwitz? S'attacherait-elle donc à dire l'indicible de cet événement, selon un geste également à l'œuvre par exemple chez Levinas ou Derrida ? Ou encore : le vide ou la dimension spatiale étrang(èr)e ("l'impression d'être dans une ville étrangère") questionnés par Perec relèveraient-ils donc de l'irruption d'une "hétérotopie" (Foucault 2009, 26), conforme à "l'événementialisme" (Balibar 2011, 213) contemporain ?

Nous ne le pensons pas. Perec nous semble donner à méditer un rapport tout à fait singulier. Si l'étrangeté qu'il questionne se soustrait au régime du normal (et de l'ordinaire), elle ne repose pas pour autant, en effet, sur le déploiement d'un excès ou d'un dehors hétérotopique émergeant dans l'ici - par exemple, la place St Sulpice - pour le transgresser. Comment Perec pourrait-il faire valoir l'irruption d'un tel au-delà événementiel alors même que le lieu épuisé devient étranger précisément quand "Il ne se passe rien, en somme" (Perec 1976, 79) ? Ou encore: comment pourrait-il donc promouvoir une épreuve de l'invisible (ou de l'indicible) alors même qu'il invite bien plutôt à "s'obliger à voir plus platement" (nous soulignons, ibid., 77) ?

Il faudrait comparer l'écriture de Perec et une autre écriture marquée par les camps de concentration : celle que déploie la phénoménologie de Levinas pour dire l'événement du visage d'autrui et l'éthique qu'il institue. Une telle comparaison conduirait probablement à marquer l'écart entre la simplicité de l'écriture du quotidien de Perec - écriture "sans style" (Schilling 2006, 180) - et le travail levinassien du langage (ou du Dit) déployé pour incessamment dédire le Dit et ainsi témoigner de l'indicibilité du visage d'autrui. Ou encore : l'écart entre la platitude de l'écriture perecquienne - adéquate à celle du quotidien - et la hauteur du dédire levinassien, nécessaire pour attester la transcendance invisible du visage.

Signalons de ce point de vue la singularité d'Ellis Island. Consacré à ce "lieu même de l'exil" qu'est Ellis Island (qui a été, pour les immigrants, le lieu principal d'entrée aux Etats-Unis au début du XXème siècle), ce texte de Perec médite le "fait même d'être juif" (Perec 2019, 61), le rattachant à "quelque chose d'informe, à la limite du dicible" (nous 
soulignons, ibid., 60-61). Dans cette perspective Perec fait valoir, sur cette île elle-même, sa démarche d'épuisement du quotidien : "Au début, on ne peut qu'essayer de nommer les choses, une à une, platement, les énumérer, les dénombrer, de la manière la plus banale possible" (nous soulignons, ibid., 45). Comme si, à nouveau, seule la platitude d'un inventaire, plutôt que la hauteur ou l'excès d'un dédire, pouvait donner accès à ce "quelque chose d'informe" - non pas indicible mais, précisément, "à la limite du dicible" - et conduire au "nulle part" (nous soulignons, Perec 2019, 22) énigmatique dont Ellis Island est également porteur selon Perec.

\subsection{Le quotidien, une platitude sans vie : Perec avec et sans Deleuze}

Nous proposons de questionner cette platitude étrange (de l'écriture) du quotidien de Perec depuis la philosophie de Deleuze et de Guattari et l'articulation qu'elle établit entre un plan d'immanence et des agencements. Un tel rapprochement doit-il être considéré comme suspect étant donné l'absence, à notre connaissance, d'échange entre DeleuzeGuattari et Perec, pourtant contemporains les uns des autres et, surtout, étant donné l'importance accordée à l'événement par la philosophie deleuzienne ?

En tous les cas, la philosophie de l'art de Deleuze privilégie ce qui, dans l'art - par exemple dans la peinture de Francis Bacon -, se refuse à la narration sans pour autant céder à l'abstraction. De ce point de vue, assez général, elle semble a priori adéquate aux textes de Perec consacrés au quotidien : si ses inventaires du quotidien refusent bien sûr toute abstraction, il est remarquable qu'hétérogènes à toute histoire, ils ne procèdent nullement d'une logique narrative.

Bien plus, Deleuze et Guattari dégagent et valorisent un plan d'existence - le plan d'immanence - qui peut être rapproché du quotidien et en particulier du quotidien médité par Perec. Du point de vue de Deleuze et de Guattari, ce plan tranche sur le plan dit d'organisation, lequel "impose [...] des organisations dominantes" (Deleuze et Guattari 2013, 197) structurées par des dualités classiques (sujet/objet, individu/collectif, etc.), au sein desquelles nous pourrions inscrire la dualité même - déjouée par Perec - du normal et de l'ordinaire (au sens de Certeau). De surcroît, plusieurs auteurs (par exemple Heidegger et Blanchot) tendent à considérer le quotidien comme un espace d'existence impersonnel, en lui-même dépourvu d'événement et soustrait à "tout début" et à "toute fin" (Blanchot 1969, 362) - autant de traits que semble bien partager le plan d'immanence comme tel établi par Deleuze et Guattari. Plus encore, Grégori Jean ainsi que Stéphane Legrand et Guillaume Silberti-Blanc (2005) ont rattaché cet espace d'existence du quotidien à un plan, comparable, selon eux, au plan d'immanence deleuzo-guattarien. L'idée même de plan 
d'immanence n'enveloppe-t-elle pas en effet un "aplatissement ontologique" (Jean 2003, 20) caractéristique de la quotidienneté ? Comme l'écrit Blanchot, "Le quotidien, c'est la platitude", avant d'ajouter : "mais cette banalité est pourtant aussi ce qu'il y a de plus important" (Blanchot 1969, 357).

Dès lors, il paraît légitime de considérer que ce plan d'immanence relève d'une platitude proche de celle qui constitue le quotidien de Perec. Soustraits aux dualités classiques, les agencements - c'est-à-dire les combinaisons "de termes hétérogènes" (Deleuze et Parnet 1996, 84) - qui peuplent ce plan d'immanence chez Deleuze et Guattari ne correspondentils pas, jusqu'à un certain point, aux occurrences que la recherche de Perec décrit ? Car chacune de ces occurrences semble bien également agencer des termes hétérogènes, par exemple : un bras de jeune fille, une raquette et une housse ou encore : des pigeons, quatre enfants, un chien, un rayon de soleil et un bus ( $\left.{ }^{\circ} 96\right)$. Que le quotidien dont Perec fait l'inventaire engage une profusion d'occurrences singulières, cela ne tiendrait-il donc pas à ce que son "tissu" (Perec 2020, 48) procède d'un plan d'immanence de type deleuzoguattarien ? Plus encore : ne pourrait-on rapprocher significativement le "bruit de fond" (Perec 1989, 11) que Perec rattache au quotidien et le "chaos" (nous soulignons, Deleuze et Guattari 1991, 189) sur lequel est branché, et à partir duquel s'établit, ce plan d'immanence ? Ne serait-ce pas là une manière de questionner le vide ou le "nulle part" que la recherche de Perec sur le quotidien donne à méditer ?

Un point déterminant sépare toutefois irréductiblement les deux perspectives : le plan d'immanence deleuzo-guattarien est un lieu d'inventivité et plus précisément de vitalité. Cette inventivité n'est pas en effet l'inventivité de l'homme ordinaire (au sens de Certeau), mais bien celle de la vie elle-même. C'est cette vie, impersonnelle, qui chez Deleuze et Guattari déborde événementiellement le plan d'organisation, le désorganise en lui soustrayant des agencements inédits, établissant ainsi des liens (entre termes hétérogènes) qui défont ses prescriptions. Pour Deleuze, c'est bien ce "surgissement" (nous soulignons, Deleuze 1996, 66) vital que doit faire valoir l'art.

Inventivité ou surgissement vital : rien de cela ne s'atteste, nous semble-t-il, dans la recherche sur le quotidien de Perec. Certes, ses descriptions convoquent des formes d'agencements, mais les liens qui les constituent - par exemple les liens entre une jeune fille, son bras, une raquette, une housse et des balles de tennis - ne sont a priori pas neufs ou innovants : un passage sur la Place St Sulpice, ce n'est pas une irruption événementielle ou un surgissement vital au sens de Deleuze ! Certes, on n'exagèrera pas ici l'écart avec l'événementialité deleuzienne dans la mesure où, loin de ne concerner que de grands événements, celle-ci détermine également ces "événements mêmes petits qui échappent au contrôle" (nous soulignons, Deleuze 2003, 239) (que l'on songe par exemple aux agence- 
ments vitaux de l'enfance qui débordent l'organisation et le contrôle d'une famille). Mais précisément : comment le simple agencement, par exemple, de quatre enfants, d'un chien, d'un petit rayon de soleil et d'un bus (le 96), pourrait-il se constituer selon une telle événementialité, échappant au contrôle ou bien désorganisant le régime du normal (ou de l'ordinaire) ? Comment cette événementialité pourrait-elle donc régir les multiples passages on ne peut plus neutres que Perec décrit place St Sulpice ? On distinguera donc ces occurrences, aussi singulières et transitoires soient-elles, des événements deleuziens dans la mesure où elles ne sauraient assumer un excès susceptible de libérer des carcans de l'organisation. Comment le reste perecquien - a priori inséparable d'une forme d'immobilité - pourrait-il être porteur du "mouvement vital" ou de la "puissance vitale" (Deleuze 1996, 31), foncièrement chaotique, qui, chez Deleuze, rend possible un tel excès ou une telle libération?

La platitude perecquienne est donc, si l'on veut, plus plate que la platitude deleuzoguattarienne, encore relevée par l'excès d'un dehors : par ce dehors - toujours événementiel - qui, conformément à la pensée contemporaine du "dehors" (Foucault 2003, 14), structure les surgissements vitaux constitutifs du plan d'immanence. Deleuze et Guattari estimeraient-ils, dès lors, que, dépourvus de tout dehors - de toute ligne de fuite comme ils le disent aussi -, les agencements perecquiens ne peuvent que demeurer prisonniers du plan d'organisation que le mouvement vital, lui, excède ? Ou doit-on bien plutôt considérer que ces agencements déjouent l'opposition même que Deleuze et Guattari établissent entre ces deux plans, dans la mesure où, aussi singuliers qu'étrangers à toute inventivité (vitale ou ordinaire), ils ne sont ni normaux/ordinaires, ni de nature à désorganiser le régime du normal (ou de l'ordinaire) ? Ni dedans, ni dehors - engageant un "nulle part" - : n'est-ce pas là en effet toute l'étrangeté du quotidien découvert par Perec ?

Peut-être ce quotidien semblera-t-il décidemment bien vide, dépourvu qu'il est, donc, non seulement de normes sociales et d'inventivité ordinaire (dedans), mais aussi de toute puissance vitale, susceptible de les excéder (dehors). Mais ne touchons-nous pas ici, précisément, à la singularité du quotidien comme tel ? Pourquoi donc faudrait-il gonfler le quotidien en le dotant d'une puissance (vitale) ou d'une force (ordinaire) qui n'est pas la sienne ? Bruce Bégout, dans La Découverte du quotidien, et Grégori Jean ont ainsi marqué, chacun à leur manière, la nécessité de ne pas charger le quotidien de ressorts - telles la créativité ou "l'intensité" (Jean 2012, 34) - qui ne sauraient le constituer. Peut-être le quotidien et sa platitude sont-ils en un sens déchargés comme peut l'être une pile, dont on dit, justement, qu'elle est 'à̀ plat'... Méditer l'énigme du quotidien, c'est bien en effet, nous semble-t-il, questionner cette dimension ni inventive, ni soumise - ni vivante, ni morte que pointent le reste perecquien et sa platitude. Puisque la philosophie contemporaine - 
qu'elle se déploie du côté de l'humain et de la transcendance (Levinas) ou de la vie et de l'immanence (Deleuze) - manque, semble-t-il, cette platitude énigmatique, continuons à la questionner à partir de certaines recherches artistiques.

\section{2. le silence du quotidien. A partir d'Hammershøi}

\subsection{L’énigme Hammershøi}

\section{Le dépouillement des intérieurs}

Nous avons découvert la peinture de Vilhem Hammershøi lors d'une exposition qui lui a été consacrée en 2019 à Paris, au Musée Jacquemart-André2. De façon générale, qu'elle soit consacrée à des paysages ruraux ou urbains, à des portraits, à des nus ou à des scènes d'intérieur, sa peinture fait preuve d'un dépouillement étonnant: les paysages témoignent d'une "absence de vie aussi bien humaine qu'animale" (Champion et Curie 2019, 106), les villes sont désertées ou en hiver, les nus dépourvus de toute sensualité.

Hammershøi a connu le succès avec ses peintures d'intérieurs : consacrées aux appartements dans lesquels le peintre lui-même vivait et aux pièces communes de ces appartements (aucune peinture ne représente une chambre ou la salle de bains), ces peintures témoignent d'une méditation originale du quotidien. Deux types de peintures d'intérieurs peuvent sans doute être distingués. Tout d'abord, dans un certain nombre de tableaux, apparaît Ida, la femme du peintre : dans la grande majorité des cas, elle est seule, souvent de dos ; à notre connaissance, dans ces intérieurs, son visage, quand on le voit, ne se présente jamais de face et semble privé de "toute psychologie ou même animation" (ibid., 78). D’autres intérieurs d'Hammershøi sont, eux, vidés "de toute présence humaine" (ibid., 154); ne demeure alors qu'"un petit théâtre d'accessoires dont Hammershøi dispose à l'envi" (ibid., 154) : des portes, des tables, des chaises, des cadres aux murs, le plus souvent dépourvus de tout ornement, motif ou détail.

Ce dépouillement est soutenu par l'usage d'une palette de couleurs particulièrement réduite, Hammershøi privilégiant souvent, en particulier au niveau des intérieurs, des tons gris, blancs et noirs, parfois bruns. Le contraste est important avec ses contemporains da-

\footnotetext{
${ }^{2}$ Notre analyse de cette peinture a significativement bénéficié de nos échanges avec Agnès Barincou.
} 
nois (comme C. Holsøe) qui, s'ils s'attachent également à peindre des intérieurs, requièrent des tons bien plus colorés et chaleureux.

La différence entre cette approche du quotidien et celle de Perec semble de prime abord importante. Dans les deux cas, nous avons certes bien affaire à un art du quotidien qui refuse le registre du narratif sans verser dans l'abstraction. Toutefois, alors que Perec s'attache à déployer un inventaire du quotidien - à décrire la profusion de ses occurrences , Hammershøi fait donc bien plutôt valoir un dépouillement singulier, comme si, loin de cibler tout "ce qui se passe", il vidait le quotidien, en particulier de toute vie et de toute interaction humaine. Pour le dire autrement: alors que les agencements perecquiens se caractérisent par leur passage (par exemple sur la place St Sulpice) et leur (relative) variété, les agencements, si l'on veut, des intérieurs d'Hammershøi - en particulier ceux qui lient sa femme à des pièces d'appartements largement vidées - se définissent davantage par leur immobilité et leur (relative) monotonie.

Mais précisément, ainsi, cette peinture semble bien rejoindre la manière dont, comme nous l'avons souligné, Perec décharge en réalité le quotidien de toute inventivité ordinaire ou vitale, ne s'intéressant à "ce qui se passe" que pour mieux découvrir qu'"Il ne se passe rien, en somme". Le quotidien immobile d'Hammershøi serait-il proche du reste de Perec ? Peut-être même pourrait-on se demander si l'importance des surfaces vides dans les peintures d'intérieurs de Hammershøi (surface des murs, des portes, des tables) ne fait pas signe, à sa façon, vers la platitude du quotidien, repérée chez Perec. En tous les cas, ces peintures semblent bien marquer, comme chez Perec, combien, vidé, le quotidien se refuse au normal (et à l'ordinaire) sans pour autant l'excéder vitalement ou événementiellement : de quel excès pourrait donc bien témoigner en effet le dépouillement - ou, comme nous le dirons, le silence - des appartements d'Hammershøi ?

\section{Une atmosphère énigmatique}

Comment donc interpréter ce dépouillement? Les commentateurs d'Hammershøi soulignent l'atmosphère dont est porteuse sa peinture. "L'absence d'un vrai sujet, d'une anecdote piquante ou d'un joli motif force le regard à s'intéresser aux vides, aux interstices, au simple poudroiement de l'air." (Curie 2019, 72). Le dépouillement de cette peinture invite ainsi à méditer les vides ou les interstices du quotidien : l'atmosphère quotidienne qui s'atteste entre les réalités ordinaires (ou normales). Comment donc questionner cette atmosphère ?

La première option est de la charger négativement. Plusieurs commentateurs marquent ainsi "l'enfermement et la solitude des êtres" (ibid., 70) - en particulier d'Ida - dont témoigneraient les tableaux d'Hammershøi, renforcés par l'absence, incontestable, dans sa peinture 
du quotidien, de tout dehors ou de toute extériorité aux intérieurs. Ainsi ces commentaires peuvent-ils évoquer un "silence oppresseur" (Claustrat 2019, 38) ou encore une "incommunicabilité" et une "condition humaine à huis clos" (ibid., 49). Nous ne partageons pas cette lecture. Pourquoi le dépouillement des peintures d'Hammershøi impliquerait-il donc une telle oppression ou un tel isolement ? Pourquoi l'absence d'interaction humaine ou de dehors signifierait-elle nécessairement un enfermement et même une solitude ? Que les intérieurs d'Hammershøi ne donnent pas sur le dehors - le dehors du monde -, cela signifie-t-il donc qu'ils constituent une forme de prison, dans laquelle Ida et le peintre lui-même seraient enfermés - ou bien plutôt qu'ils découvrent la dimension du quotidien, en tant qu'elle se soustrait à l'ordinaire (ou au normal) constitutif du monde ?

De même qu'il ne nous paraît pas légitime de gonfler le quotidien de Perec de ressorts a priori positifs - telle l'inventivité -, il ne nous paraît pas légitime de charger le quotidien d'Hammershøi de ressorts négatifs - tels des affects de solitude ou d'enfermement. Que ses peintures ne témoignent pas de l'atmosphère de douceur propre à la peinture de certains de ses contemporains danois - qu'elles ne contiennent aucune éloge du quotidien (Todorov, 1997) - est une chose, qu'elles attestent pour autant un enfermement ou une incommunicabilité en est une autre. Peut-être faudrait-il ici convoquer l'idée de neutre - et même d'"énigme du neutre" (Blanchot 2008, 97) - proposée par Blanchot. La palette ni colorée, ni obscure, privilégiée par la peinture d'Hammershøi, ne suggère-t-elle pas en effet cette neutralité énigmatique d'un quotidien ni inventif, ni enfermant - neutralité qu'évoque peut-être aussi Perec quand il revendique, à propos de sa recherche sur le quotidien, une description qui "se veut la plus neutre possible" (Perec 1976, 83)?

En tous les cas, d'autres commentaires de cette peinture insistent bien, en effet, sur son caractère énigmatique et mystérieux, soulignant ainsi, par exemple, que "Le mystère est" sa "marque de fabrique" (Claustrat 2019, 45) ou encore que, dans ses intérieurs, Hammershøi oppose un "mystère métaphysique" au "confort domestique" (Champion et Curie 2019, 95) caractéristique des intérieurs de certains de ses contemporains danois. Comment donc questionner cette atmosphère énigmatique identifiée comme telle par Harri Mäcklin (2018) ?

\subsection{Le quotidien, un silence sans nuit : Hammersh øi avec et sans Levinas}

Mäcklin propose d'approcher la peinture d'Hammershøi et tout particulièrement l'atmosphère de ses intérieurs avec le concept d'il y a de Levinas, concept proche de l'idée blanchotienne de neutre. Cette proposition est intéressante à plusieurs titres.

Tout d'abord, l'il y a engage bien, dans la phénoménologie de Levinas, une pensée atmosphérique de l'art. En effet, de son point de vue, l'art doit être rattaché à cette "atmos- 
phère" (Levinas 1990, 104) non localisée - relevant d'un "nulle part" (Levinas 1992, 151) que constitue précisément l'il y a. A cette dimension atmosphérique de l'art, Levinas oppose, pour le privilégier, le face-à-face éthique avec le visage d'autrui. Conséquence importante : si elle se refuse bien au normal ou à l'ordinaire - tous deux relevant chez Levinas du régime du Même -, l'atmosphère de l'il y a ne s'y refuse pas à la manière d'un au-delà événementiel, réservé à l'éthique du visage. Une telle dimension atmosphérique, ni ordinaire, ni événementielle - ni Même, ni Autre -, propre à l'art, et plus spécifiquement à la peinture, semble également s'attester dans la dernière phénoménologie de Merleau-Ponty (à l'œuvre dans L'Eil et l'Esprit).

La phénoménologie levinassienne de l'art engage donc bien, ainsi, la méditation d'une atmosphère singulière, et même d'une atmosphère silencieuse. Insistons sur ce "silence" (Levinas 1990, 95) de l'il y a dans la mesure où il paraît tout à fait adéquat aux peintures d'Hammershøi. Dans la lignée du propos levinassien, on pourrait ainsi opposer ce silence atmosphérique à la parole du visage d'autrui ou du face-à-face avec autrui, tout à fait absente des intérieurs d'Hammershøi. Il est remarquable de ce point de vue qu'Ida, la femme du peintre, y soit toujours représentée soit de dos, soit de biais ; remarquable également que, dans Double portrait de l'artiste et de sa femme, à travers un miroir (1911), les deux époux se tournent le dos. Hammershøi ne marque-t-il pas ici le silence qui peut s'attester entre - et autour de - nous?

Enfin, dans "La Réalité et son ombre" - texte où Levinas dénonce l'ombre attaché à l'art (et à l'il y a) ou encore "le commerce avec l'obscur" (nous soulignons, Levinas 1994, 126) qui le constitue -, le philosophe pense la temporalité de cette atmosphère silencieuse comme une interruption du temps ou une immobilité, évoquant également une "vie sans vie" ou encore une "caricature de vie" (Levinas 1994, 139). Levinas ne fait-il pas ici signe vers l'immobilité des intérieurs d'Hammershøi et, plus encore, vers l'insistance du quotidien en tant qu'elle est dénuée de toute durée créative (au sens de Bergson) ou de toute puissance vitale, ainsi que nous l'avons suggéré à partir de Perec ?

Ce rapprochement pose toutefois problème. L'il y a est en effet chargé par Levinas d'une "densité" (Levinas 1990, 104) lestée d'un affect négatif : l'atmosphère de l'il y a est porteuse d'une "lourde ambiance" (ibid., 95). Faut-il dès lors, comme le suggère Mäcklin, accentuer encore la pertinence de l'il y a au regard de la peinture d'Hammershøi dans la mesure où, comme nous l'avons indiqué, celle-ci témoigne également, selon certains de ses commentateurs, d'une atmosphère pesante ou oppressante ? En réalité, cette densité ou lourdeur de l'il y a tient d'abord à ce qu'il se constitue, chez Levinas, comme une nuit : son atmosphère procède en effet d'un "espace nocturne" (ibid., 95) ou encore d'un "fond obscur" (nous soulignons, ibid., 98). Plus encore, ce fond constitutif de l'il y a implique une 
épreuve de l'obscur : une "dissolution du sujet dans la nuit" (ibid., 103) - épreuve ou dissolution de l'il y a dont il est remarquable qu'elles demeurent, malgré tout, événementielles (Levinas insistant en effet sur "l'événement impersonnel de la nuit et de l'il y $a$ ", ibid., 104). Or, rien de cela ne s'atteste, de notre point de vue, dans la peinture du quotidien d'Hammershøi. Si, dans ses intérieurs, Ida n'est jamais en situation de répondre à autrui, elle ne se dissout pas pour autant dans un fond obscur et "indistinct" (ibid., 99), de même que ne s'y dissolvent pas les portes, les cadres, les meubles: tout ce "petit théâtre d'accessoires" dont joue Hammershøi. Par exemple, dans Intérieur, Strandgate 30 (1899), le visage de Ida, penché, les yeux baissés, est peint avec un blanc également requis pour sa jupe, deux portes, une tasse et une cafetière, l'ensemble témoignant d'une atmosphère aussi silencieuse que dépourvue de toute lourdeur ou pesanteur, un commentateur parlant même d'une "sensation de paix" (Champion et Curie 2019,138). Comment pourrait-on penser l'atmosphère d'un tel tableau depuis la nuit impersonnelle ou depuis l'épreuve de l'il y a ?

On distinguera donc bien le dépouillement de ses peintures et la dissolution opérée par l'il y a, d'autant que Levinas évoque également, à propos de l'il y a, un "mouvement qui va dépouiller" (nous soulignons, Levinas 1990, 98) la subjectivité. Que les intérieurs soient vidés de toute interaction humaine n'implique pas pour autant qu'ils reconduisent à un fond obscur impersonnel. De même que les agencements perecquiens, ni normaux/ordinaires, ni événementiels, déjouent les catégories deleuziennes, le silence d'Hammershøi ne procède donc, précisément, d'aucune des trois dimensions qui structurent la phénoménologie levinassienne : le Même (normal/ordinaire), l'Autre (événementiel) et l'il y a. Au-delà même de Levinas, il faudrait se demander si ce silence atmosphérique - ni personnel, ni impersonnel - peut encore être considéré comme un fond ou une radicalité de type phénoménologique. De quelle force de type transcendantal ou de quelle puissance radicale pourrait donc en effet être chargé le silence d'Hammershøi, alors même que sa peinture semble déchargée - dépouillée - non seulement de toute humanité (ou vitalité), mais également de toute puissance nocturne porteuse d'in-humanité (ou de mort) ? Faudrait-il opposer, dans cette perspective, l'insistance du quotidien peint par Hammershøi et la consistance des atmosphères radicales établies par les phénoménologies de Levinas ou de Merleau-Ponty, dont le silence est encore déterminé par la puissance d'une "voix" (ibid., 95) ?

En tous les cas, ni fort, ni faible, le silence d'Hammershøi semble donc, lui aussi, porteur d'un rapport bien étrange. Car, aussi dépouillé de l'ordinaire soit-il, ce silence ne paraît pas plus s'établir en deçà de l'ordinaire, dans un fond obscur, qu'il ne se déploie événementiellement au-delà. Le dépouillement de cette peinture ne touche-t-il pas ainsi à la simplicité même du quotidien, en tant qu'elle se refuse à la complexité de l'ordinaire sans 
pour autant convoquer la radicalité phénoménologique (en deçà de l'ordinaire) ou l'événementialité contemporaine (au-delà de l'ordinaire) ?

\section{Un art du commun?}

Platitude sans vie, silence sans nuit : l'art du quotidien proposé par Perec et Hammershøi consisterait-il donc à vider le quotidien de toute épaisseur et, finalement, de toute signification? Serait-il donc nihiliste ? Nous ne le pensons pas : il faut bien plutôt questionner positivement la dimension que ces deux artistes libèrent ainsi du primat de la vie ou de l'épreuve de l'obscur. Bien sûr, on se gardera d'identifier hâtivement Perec et Hammershøi et de confondre la platitude ou le "nulle part" du premier et le dépouillement ou le silence du second. Toutefois, tous deux font signe vers un art du quotidien, dont nous proposons d'amorcer en conclusion la conceptualisation.

Inventaire de "choses communes" conduisant à l'étrangeté d'un vide ou d'un "nulle part" ; dépouillement d'un silence s'attestant dans les pièces communes d'un appartement : dans ces deux recherches, le quotidien semble, tout d'abord, engager un rapport bien singulier, conjuguant un ancrage irréductible dans le "proche" (Champion 2019, 28) et l'ordinaire - dont relèvent ces choses et ces pièces - et une dimension pour le moins énigmatique, tout à fait non ordinaire : un "nulle part", un silence. Notre hypothèse est que la philosophie continentale contemporaine, en particulier quand elle pense l'art, n'est pas en mesure d'établir ce rapport soit parce qu'elle privilégie un au-delà de type événementiel (comme chez Deleuze et dans la pensée du dehors), soit parce qu'elle fait valoir un en deçà fondateur (comme chez Levinas et en phénoménologie) - ces deux gestes reconduisant, semble-t-il, une épreuve de l'invisible ou de l'obscurité (le chaos de Deleuze, il y a de Levinas). Ni au-delà, ni en deçà de l'ordinaire : le quotidien n'enveloppe-t-il pas un à même l'ordinaire pour le moins étrange, que seul un certain art, affranchi de cette épreuve, est susceptible de méditer? Un certain art qui, à la différence de la philosophie, questionne le quotidien - son étrangeté - sans le gonfler ou le charger d'événement ou d'affect, de vie ou d'(in)humanité ?

De surcroît, dans les deux cas, cet art du quotidien fait signe vers une dimension collective inédite. Nous l'avons souligné : Perec valorise les choses communes et cherche même, par sa méditation du quotidien - d'une place, d'une rue, d'un escalier d'immeuble, d'un "appartement" (Perec 1976, 37) et de ses pièces... -, à jeter "les bases d'une existence collective" (ibid., 67). Ne rejoint-il pas ici la recherche d'Hammershøi, dont la peinture accorde tant d'importance aux portes ou aux chaises des pièces communes de ses intérieurs ? Hammershøi donne ainsi à penser, nous semble-t-il, l'entre nous du quotidien, cette 
première personne du pluriel (entre nous) signifiant combien l'atmosphère silencieuse qu'il médite s'atteste entre lui-même et sa femme, au sein même des pièces qu'ils habitent peut-être aussi entre eux et nous, qui sommes invités à méditer ces intérieurs dépouillés ? Cet entre nous n'est-il pas également suggéré par Perec dès lors que son geste propre d'inventaire des agencements - sans vie - du quotidien se déploie "sur le lieu même" (ibid., 83) du quotidien (par exemple place St Sulpice) ? Cet art du quotidien échappe peut-être à la philosophie contemporaine parce qu'il déjoue ainsi, à sa façon, la question du sujet - si importante du point de vue de cette philosophie -, refusant, en effet, de promouvoir une nouvelle force (ou faiblesse) subjective (ou humaine) sans pour autant établir une puissance impersonnelle (vitale ou nocturne) susceptible de le déconstruire (impersonnalité qui ne pourrait en effet que contrevenir à l'entre nous). Peut-être s'agirait-il ainsi, finalement, d'établir un art du commun, du moins si le commun peut conjuguer la simplicité - ni radicale, ni événementielle - du quotidien et cette dimension collective - ni (in)humaine, ni vitale - de l'entre nous.

Prof. Dr. Hugues Choplin, Université de Technologie de Compiègne, hugues.choplin[at]utc.fr

\section{Références}

Badiou, Alain. Conditions. Paris: Seuil, 1992.

Balibar, Etienne. "La Philosophie et l'Actualité : au-delà de l'événement ?" in Patrice Maniglier (ed.). Le Moment philosophique des années 1960 en France. Paris: PUF, 2011. 211-234.

Bégout, Bruce. La Découverte du quotidien. Paris: Allia, 2005.

Blanchot, Maurice. L'Entretien infini. Paris: Gallimard, 2004.

Blanchot, Maurice. Le Pas au-delà. Paris: Gallimard, 2008.

Champion, Jean-Loup. "Hammershøi et les siens ou l'intérieur de Hammershøi" in Jean-Loup Champion et Pierre Curie (eds.). Hammersh $\phi i$. Le maître de la peinture danoise. Bruxelles: Fonds Mercator, Culturespaces, Musée Jacquemart-André. Institut de France, 2019. 18-35.

Champion, Jean-Loup et Pierre Curie (eds.). Hammersh $\phi$. Le maître de la peinture danoise. Bruxelles: Fonds Mercator, Culturespaces, Musée Jacquemart-André. Institut de France, 2019.

Claustrat, Franck. "Quand le silence est d'or : Vilhem Hammershøi, Peter Ilsted, Carl Holsøe..." in Jean-Loup Champion et Pierre Curie (eds.). Hammersh $\phi i$. Le maître de la peinture danoise. Bruxelles: Fonds Mercator, Culturespaces, Musée Jacquemart-André. Institut de France, 2019. 36-51. 
Curie, Pierre. "L'âme du vide. Quelques antécédents hollandais de l'art de Hammershøi" in JeanLoup Champion et Pierre Curie (eds.). Hammershøi. Le maître de la peinture danoise. Bruxelles: Fonds Mercator, Culturespaces, Musée Jacquemart-André. Institut de France, 2019. 66-73.

Deleuze, Gilles. Francis Bacon. Logique de la sensation. Paris: Editions de la différence, 1996.

Deleuze, Gilles. Pourparlers, 1972-1990. Paris: Minuit, 2003.

Deleuze, Gilles, et Felix Guattari. Qu'est-ce que la philosophie ? Paris: Minuit, 1991.

Deleuze, Gilles, et Claire Parnet. Dialogues. Paris: Flammarion, 1996.

Foucault, Michel. La Pensée du dehors. Cognac: Fata Morgana, 2003.

Foucault, Michel. Le Corps utopique, les Hétérotopies. Paris: Lignes, 2009.

Jean, Grégori. "Deleuze et le problème de la vie - vers une ontologisation du quotidien". Revue du Céniphé $\mathrm{n}^{\circ} 3$, 2003, http://ceniphe.free.fr/revue3_files/G.Jean-Deleuze.pdf.

Jean, Grégori. Le Quotidien en situations. Enquête sur les phénomènes sociaux. Louvain-la Neuve: Presses universitaires de Louvain, 2012.

Le Blanc, Guillaume. Les Maladies de l'homme normal. Paris: Vrin, 2007.

Legrand, Stéphane et Guillaume Sibertin-Blanc. "Politiques du quotidien", 2 mars 2005, https://philolarge.hypotheses.org/files/2017/09/02-03-2005_legrand_sibertin.pdf.

Levinas, Emmanuel. De l'existence à l'existant. Paris: Vrin, 1990.

Levinas, Emmanuel. Totalité et Infini. Essais sur l'extériorité. Paris: Le Livre de Poche, 1992.

Levinas, Emmanuel. Les Imprévus de l'histoire. Cognac: Fata Morgana, 1994.

Macherey, Pierre. "Perec chroniqueur de l'infra-ordinaire", 26/01/2005, https://philolarge.hypotheses.org/ files/2017/09/26-01-2005.pdf.

Mäcklin, Harri. "How to paint nothing? Pictorial Depiction of Levinasian il y a in Vilhem Hammershøi's Interiorior paintings." Journal of Aesthetics and Phenomenology 5:1 (2018): 15-29.

Perec, Georges. Espèces d'espaces. Journal d'un usager de l'espace. Paris: Denoël/Gonthier, 1976.

Perec, Georges. L'Infra-ordinaire. Paris: Seuil, 1989.

Perec, Georges. Penser/Classer. Paris: Seuil, 2003.

Perec, Georges. Ellis Island. Paris: P.O.L, 2019.

Perec, Georges. Tentative d'épuisement d'un lieu parisien, Paris: Christian Bourgeois éditeur, 2020.

Saison, Maryvonne. "Le tournant esthétique de la phénoménologie." Revue d'Esthétique, n³6 (1999): 125-140.

Schilling, Derek. Mémoires du quotidien : les lieux de Perec. Villeneuve d'Ascq: Presses Universitaires du Septentrion, 2006.

Todorov, Tzvetan. Eloge du quotidien. Essai sur le peinture hollandaise du XVII siècle. Paris: Seuil, 1997.

Yvan, Frédéric. "L'Extase du vide de Un Homme qui dort à Espèces d'espaces de Georges Perec". Savoirs et clinique, vol. 8, no. 1, 2007, 143-153. 